\title{
Exceptional points and the topology of quantum many-body spectra
}

\author{
David J. Luitz $\mathbb{1}^{*}$ and Francesco Piazza ${ }^{\dagger}$ \\ Max Planck Institute for the Physics of Complex Systems, Noethnitzer Str. 38, 01187 Dresden, Germany
}

(Received 28 June 2019; published 28 October 2019)

\begin{abstract}
We show that in a generic, ergodic quantum many-body system the interactions induce a nontrivial topology for an arbitrarily small non-Hermitian component of the Hamiltonian. This is due to an exponential-in-systemsize proliferation of exceptional points which have the Hermitian limit as an accumulation (hyper)surface. The nearest-neighbor level repulsion characterizing Hermitian ergodic many-body systems is thus shown to be a projection of a richer phenomenology, where actually all the exponentially many eigenvalues are pairwise connected in a topologically robust fashion via exceptional points.
\end{abstract}

DOI: 10.1103/PhysRevResearch.1.033051

\section{INTRODUCTION}

Exceptional points are a particular type of spectral degeneracy where groups of complex eigenvalues coalesce as well as the corresponding eigenvectors. This is caused by an underlying square-root singularity, leading to nontrivial topological features [1].

Exceptional points play a crucial role in the understanding of topological phases in non-Hermitian bands [2-12], both in one [13-25] and higher [26-36] spatial dimensions. This topic is very recently receiving large attention, since a direct physical application to dissipative systems [37] with controlled experimental platforms also became available [38-51]. So far, exceptional points have been explored in situations where they arise in single-particle bands. While the addition of interactions can lead to novel collective phenomena [52], the emergence of exceptional points for many-body bands remained so far unexplored.

Here we provide an example in quantum many-body systems and demonstrate that exceptional points actually appear generically. We namely show that in an ergodic quantum many-body system the interactions induce a nontrivial topology for an arbitrarily small non-Hermitian component of the Hamiltonian. This is due to an exponential-in-system-size proliferation of exceptional points which have the Hermitian limit as an accumulation (hyper)surface. The connection between level repulsion in the Hamiltonian spectrum of an ergodic system and the distribution of exceptional point has been so far argued based on toy models [53] and demonstrated at fine-tuned points of models without local degrees of freedom which become classical in the thermodynamic limit $[54,55]$. Here we demonstrate such a scenario in fully

\footnotetext{
*dluitz@pks.mpg.de

†piazza@pks.mpg.de
}

Published by the American Physical Society under the terms of the Creative Commons Attribution 4.0 International license. Further distribution of this work must maintain attribution to the author(s) and the published article's title, journal citation, and DOI. generic local many-body systems with no semiclassical limit and without any fine tuning of microscopic parameters.

A remarkable and defining feature of generic ergodic many-body system follows from the exponential proliferation of exceptional point arbitrarily close to the Hermitian limit: The many-body spectrum can be understood as a single Riemann surface, where all eigenvalues are adiabatically connected along smooth paths, as any of the $N(N-1) / 2$ possible pairs of eigenvalues can be interchanged by encircling the corresponding exceptional points. Here, $N=\operatorname{dim}(\mathcal{H})$ is the dimension of the Hilbert space which grows exponentially with the system size $L$ of the quantum many-body system.

\section{MODEL}

In order to demonstrate the above scenario we consider a quantum spin- $\frac{1}{2}$ chain, described by the Hamiltonian

$$
\begin{aligned}
\hat{H}(z)= & \sum_{i=1}^{L-1} J_{x} \hat{\sigma}_{i}^{x} \hat{\sigma}_{i+1}^{x}+J_{y} \hat{\sigma}_{i}^{y} \hat{\sigma}_{i+1}^{y}+J_{z} \hat{\sigma}_{i}^{z} \sigma_{i+1}^{z} \\
& +\vec{h}_{1} \cdot \hat{\vec{\sigma}}_{1}+\vec{h}_{L} \cdot \hat{\vec{\sigma}}_{L} \\
& +z \sum_{i=1}^{L}\left(g_{x z} \hat{\sigma}_{i}^{x} \hat{\sigma}_{i+1}^{z}+g_{x y} \hat{\sigma}_{i}^{x} \hat{\sigma}_{i+1}^{y}+g_{y z} \hat{\sigma}_{i}^{y} \hat{\sigma}_{i+1}^{z}\right),
\end{aligned}
$$

with fixed real parameters $J_{x}, J_{y}, J_{z}, g_{x z}, g_{x y}, g_{y z} \in \mathbb{R}$ and fixed real boundary fields $\vec{h}_{1}, \vec{h}_{L} \in \mathbb{R}^{3}$. The site indices are defined modulo $L$, where needed [last sum in Eq. (1)]. With these choices, the Hamiltonian is a matrix-valued function of one scalar complex parameter $z \in \mathbb{C}$. For all $z$ on the real axis, the Hamiltonian is Hermitian, while it is non-Hermitian in general. Throughout this paper we take the generic choice of parameters $J_{x}=1.2, J_{y}=1.0, J_{z}=0.7, g_{x z}=0.91, g_{x y}=$ $0.7, g_{y z}=1, \vec{h}_{1}=(0.0291241,0.02341097,0.0567)^{T}, \vec{h}_{L}=$ $(0.091241,0.018924,0.0781652)^{T}$, such that the Hamiltonian has no symmetries. Note that this parameter set is not fine tuned and that we find the same phenomenology with other parameter choices. The role of the boundary fields is to destroy the reflection symmetry of the Hamiltonian as well as the integrability of the Hamiltonian at $z=0$. 
We have verified that the Hamiltonian for $z \in \mathbb{R}$ is ergodic in the sense that local observables thermalize by means of the eigenstate thermalization hypothesis [56-60], which is valid in this system (cf. Appendix A). Furthermore, we have considered the statistics of level spacings of the Hermitian Hamiltonian, using the ratio of adjacent gaps [61]. We find that the spectral statistics are in the Gaussian unitary ensemble (GUE) universality class (cf. Appendix A).

Before proceeding with the results, a comment on the implementation of the model is in order. One can consider an open system described (in a Markovian approximation) by a quantum master (Lindblad) equation, governing the time evolution of the density matrix of the system. This dynamics can be mapped onto a stochastic Schrödinger equation, where the evolution is governed by a non-Hermitian Hamiltonian, plus the action of so-called quantum "jumps" at random times. The rate of the jumps is set by the same constant setting the size of the non-Hermitian component of the Hamiltonian, this constant being related to the effective system-bath coupling [62-64]. In the limit of a small non-Hermitian component which will be considered here, since the jump rate will be similarly small, it should be experimentally feasible to determine whether a jump has occurred and to discard such runs in the analysis (so-called post-selection [50,51,65]), which leaves us with an experimental implementation of purely non-Hermitian Hamiltonian dynamics. In order to implement the specific non-Hermitian term in our Hamiltonian (1), which involves multiple spins, the correlation length in the bath should amount to at least two lattice sites, which makes the Kossakowski matrix in the Lindblad equation nondiagonal.

\section{PROLIFERATION OF EXCEPTIONAL POINTS}

Since exceptional points necessarily are related to a degeneracy of (at least) two eigenvalues of $H(z)$, it is natural to consider the distances between all eigenvalues $\lambda_{i}$ of $H(z)$. We show in Fig. 1 the minimal distance of all pairs of eigenvalues $\delta(z)=\min _{i, j}\left|\lambda_{i}(z)-\lambda_{j}(z)\right|$ as a function of the complex interaction parameter $z$. If this distance $\delta(z)$ vanishes, it corresponds to a degeneracy of at least two eigenvalues. While $\delta(z)$ is a continuous function of $z$, it is not analytic, and exhibits a large number of kinks, when the closest pair of eigenvalues changes.

We observe that as a function of system size the number of very small eigenvalue distances increases significantly. It is also clear that the minima of $\delta(z)$ appear to be very sharp, consistent with the typical square-root singularity of exceptional points. The extreme proliferation of degeneracies seems to occur most strongly close to the real axis, when $\operatorname{Im}(z)=0$.

While the results of Fig. 1 are already a strong indication of the proliferation of exceptional points and their accumulation on the real axis $\operatorname{Im}(z)=0$, in order to have a solid quantitative characterization we need to resort to the defining feature of exceptional points: the occurrence of a square-root branching point, distinguishing them from other possible degeneracies [66]. This leads to the swapping of a pair (or more in the case of higher-order exceptional points, which we do not observe here) of eigenvalues when following a closed path around an

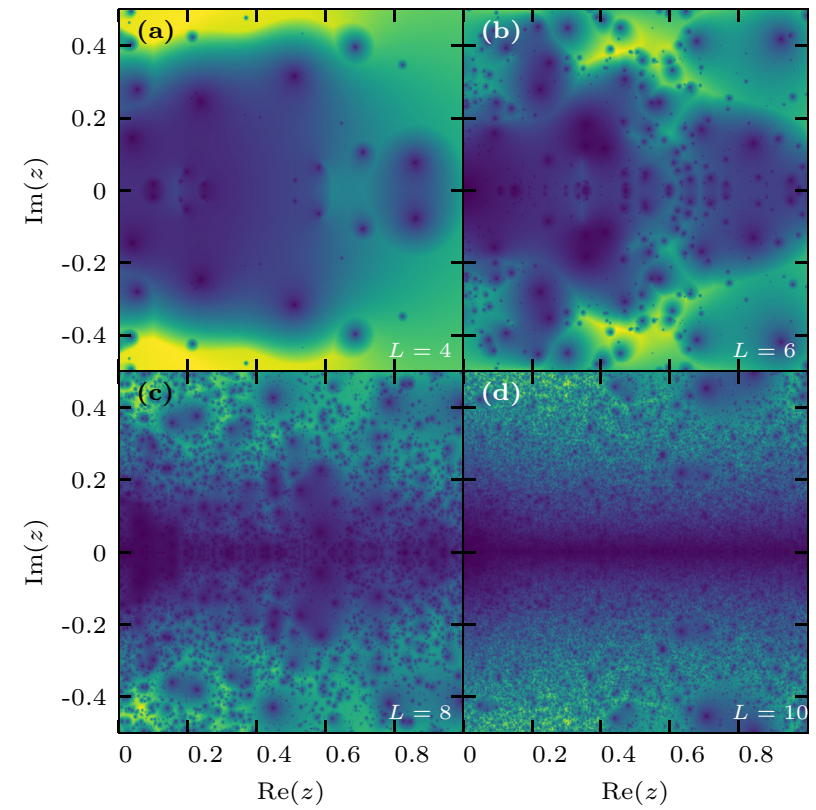

FIG. 1. Minimal eigenvalue distance $\min _{i, j}\left|\lambda_{i}(z)-\lambda_{j}(z)\right|$ between all eigenvalues $\lambda_{i}(z)$ of the Hamiltonian (1) as a function of the complex parameter $z$ for different lengths $L$ of the spin chain. The sharp minima (dark colors) correspond to degeneracies of the spectrum, which we identify to be exceptional points associated with a square-root singularity of the eigenvalue distance.

exceptional point. We will extensively use this property to study the density of exceptional points with respect to the distance from the real axis in parameter space.

\section{BRAIDING ON A SINGLE RIEMANN SHEET}

In Fig. 2 we demonstrate that the degeneracies appearing in Fig. 1 are indeed associated with exceptional points. Figure 2(a) contains a color map of the minimal eigenvalue distance $\min _{i, j}\left(\left|\lambda_{i}-\lambda_{j}\right|\right)$ as in Fig. 1 for a chain of length $L=6$. The location of degeneracies in the spectrum is clearly visible in dark spots. The rectangular and elliptical paths drawn in Fig. 2(a) correspond to example paths we consider, encircling different numbers of exceptional points. Next to each path, we list the number of transpositions in the permutation of eigenvalues after closure of the path. This is achieved by tracking the eigenvalues along the path as described in Appendix B and afterward analyzing the resulting permutation as described in Appendix C. Within one half plane of the complex plane, these numbers correspond to the number of encircled exceptional points, while exceptional points at conjugate locations $\left(z\right.$ and $\left.z^{*}\right)$ have opposite handedness and therefore undo swaps mutually. We note here that in our system each exceptional point has a conjugate partner since $H\left(z^{*}\right)=H(z)^{\dagger}$, and the spectra of $H$ and $H^{\dagger}$ are identical (since this corresponds to the adjoint eigenvalue problem). The swapping of eigenvalues (crosses) $\lambda_{i}$ is exemplified in Fig. 2(b), where each colored line corresponds to the trajectory in the complex eigenvalue space of one eigenvalue along one traversal of the path labeled by $\mathcal{P}$ in Fig. 2(a). It is apparent that the four exceptional points enclosed by the path swap two pairs of eigenvalues, 


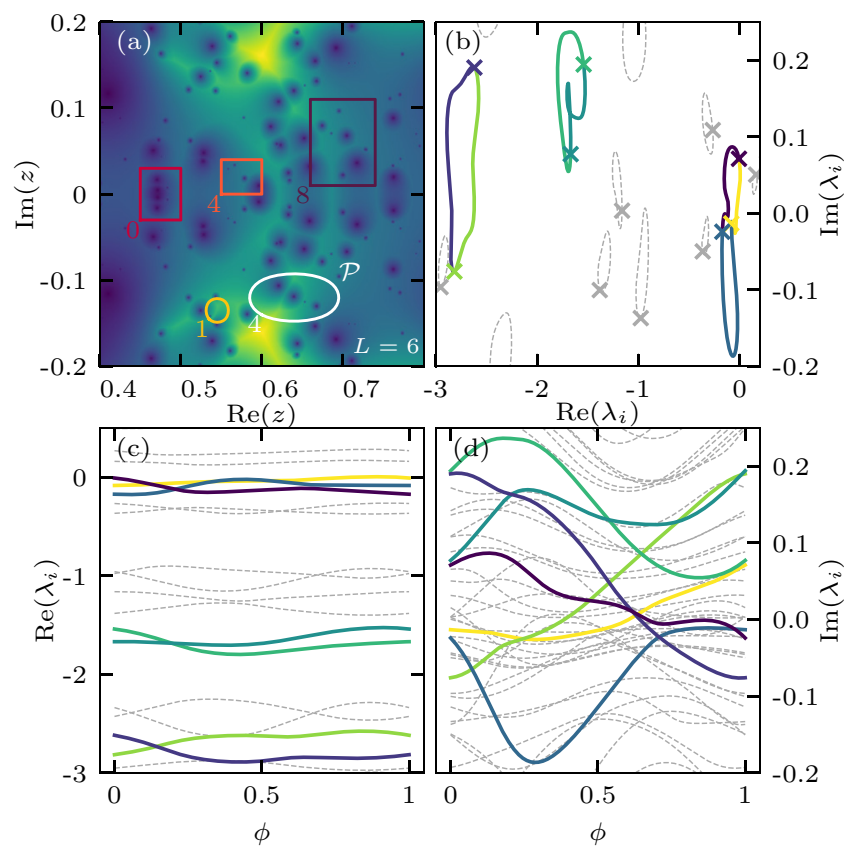

FIG. 2. Braiding of eigenvalues $E_{n}(z)$ along closed paths $g(\phi)$ : $[0,1] \rightarrow \mathbb{C}$ with $g(0)=g(1)$, encircling exceptional points for a chain of length $L=6$. (a) Overview of minimal eigenvalue distances $\min _{i, j}\left(\left|\lambda_{i}-\lambda_{j}\right|\right)$ as a function of the complex parameter $z$. Closed curves are examples with the number indicating the number of transpositions in the permutation linking the final eigenvalues $\lambda_{i}(1)$ with their initial order $\lambda_{i}(0)$. (b) For the white path $\mathcal{P}$ in (a), we show the evolution along the curve of the relevant eigenvalues (colored). Lower panels: eigenvalue evolution [real (c) and imaginary (d) parts] $\lambda_{i}(\phi)$ along $\mathcal{P}$.

and permute three more eigenvalues in a cycle corresponding to two transpositions.

Any conjugate pair of exceptional points in the complex parameter plane is connected by a branch cut, interconnecting the Riemann sheets on which each eigenvalue evolves. Due to the proliferation of exceptional points, the Riemann surface of our ergodic quantum many-body model becomes massively interconnected. As we shall see next, the proliferation is exponential in the system size $L$ such that there is one exceptional point for each possible pair of eigenvalues. This means that starting from one eigenvalue $\lambda_{i}$, any other eigenvalue can be reached by adiabatic parameter changes in the complex plane, that is, the spectrum of an ergodic quantum many-body system actually belongs to a single Riemann surface.

\section{STATISTICS OF EXCEPTIONAL POINTS}

To characterize the proliferation of exceptional points with increasing system size, we compute their distribution in the complex plane $\operatorname{Re}(z), \operatorname{Im}(z)$. In Fig. 3 we show the density of exceptional points as a function of the distance $\operatorname{Im}(z)$ from the real axis. We compute the density $\rho_{\mathrm{EP}}=M / A$ by counting the number $M$ of exceptional points within a given area $A$ in the complex plane (as described above). In order to collect enough statistics still keeping computational times feasible, we choose the area $A$ such that it typically contains between 100 and 1000 exceptional points in the bulk of the distribution,
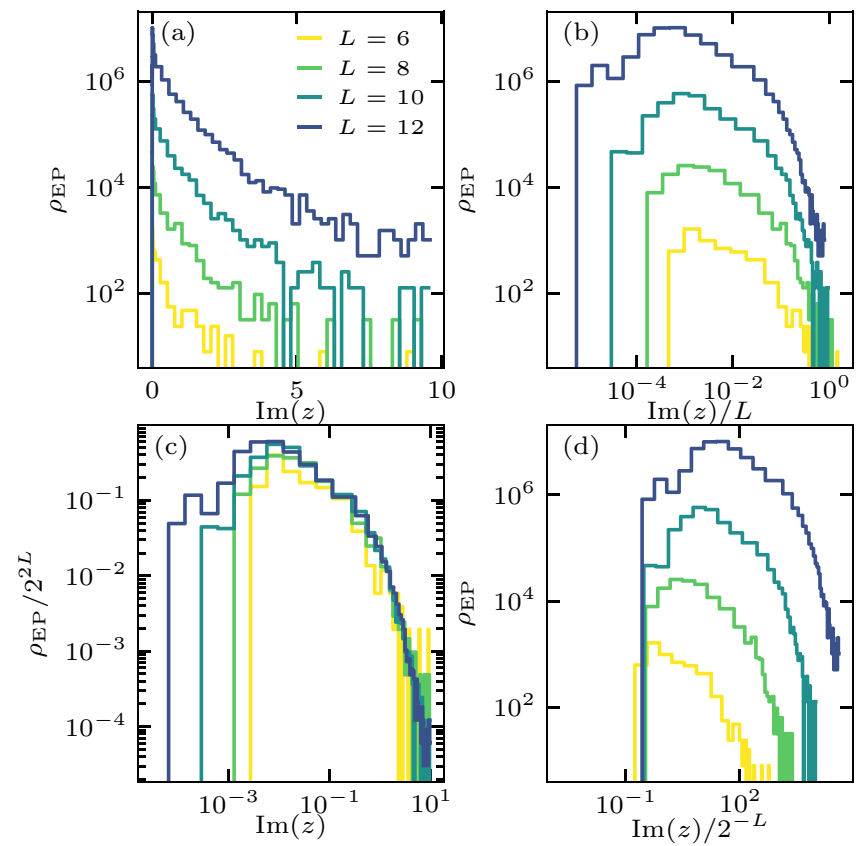

FIG. 3. Density $\rho_{\mathrm{EP}}$ of exceptional points per unit area in the complex parameter plane. We consider parameter areas at $\operatorname{Re}(z)=$ 0.5 and count the number of exceptional points in areas of size $d \operatorname{Re}(z) d \operatorname{Im}(z)$, with exponentially decreasing bin size $d \operatorname{Re}(z) \propto$ $2^{-L}$. (a) Density versus distance from the real axis in parameter space for different system sizes. (b) Density versus distance from the real axis rescaled by $1 / L$. (c) Density rescaled by the total number of exceptional points $2^{2 L}$. (d) Density versus distance rescaled by $1 / 2^{-L}$.

independently of the system's size (which as we shall see implies exponential downscaling of $A$ with $L$ ). As apparent from Fig. 1, the density becomes more and more independent of the position on $\operatorname{Re}(z)$ as $L$ is increased. Selecting areas $A$ of a finite width along $\operatorname{Re}(z)$, we effectively average over $\operatorname{Re}(z)$ to improve the statistics.

Figure 3(a) shows the density up to large distances from the real axis, so that the tails of the distribution (compatible with an exponential decay) are visible. More interesting is the distribution in the vicinity of the real axis, as shown in Figs. 3(b) 3(d). In Fig. 3(b), we see that the distribution extends up to distances from the real axis which scale with the system size $L$. Moreover, as apparent from Fig. 3(c), the overall scale of the density increases exponentially as $2^{2 L}$. Taking into account the fact that the tails of the distribution extend to values of $\operatorname{Im}(z)$ of the order of $L$, this scaling is consistent with having a proliferation of exceptional points such that we have one for each possible pair of eigenvalues of the Hamiltonian (1), i.e., $N(N-1) / 2$ with $N=\operatorname{dim}(\mathcal{H})=2^{L}$. Finally, Fig. 3(d) shows that the bottom of the distribution has a gap from the real axis which vanishes like $2^{-L}$, which demonstrates the exponentially fast accumulation of exceptional points at the Hermitian line $\operatorname{Im}(z)=0$. This scaling of the gap is to be expected under two assumptions: (i) the exceptional points at the bottom of the distribution are the ones connected to the avoided crossings between nearest-neighbor levels of the Hermitian Hamiltonian $\operatorname{Im}(z)=0$ (see discussion below 


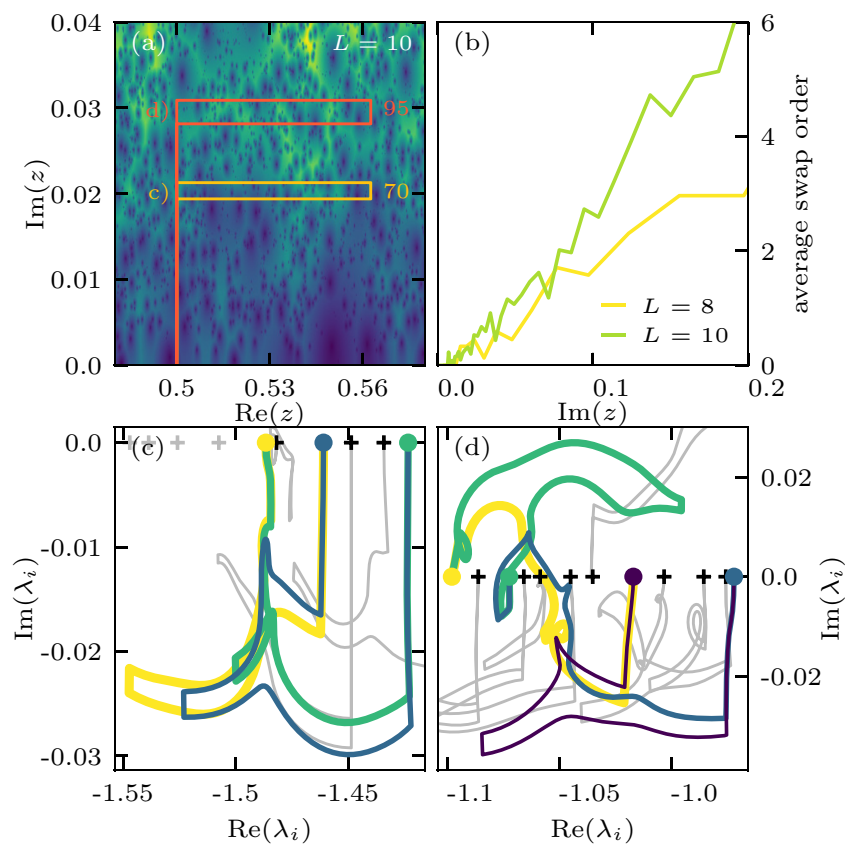

FIG. 4. (a) Zoomed overview of the minimal eigenvalue distance $\min _{i, j}\left|\lambda_{i}-\lambda_{j}\right|$ for a system of size $L=10$. Solid lines are the two exemplary paths connected to the real parameter axis for which eigenvalue traces are shown in (c) and (d). (b) Average distance of swapped eigenvalues of the Hermitian system by exceptional points as a function of their distance from the real axis for $L=8$ and 10 . (c), (d) Exemplary eigenvalue cluster traces swapped (colored) by exceptional points encircled by the paths shown in (a).

and Fig. 4); (ii) the distance of each exceptional point from the real axis is proportional to the real gap characterizing the corresponding avoided level crossing. Assumption (ii) is actually a mathematical fact for a single exceptional point but not trivial in the presence of multiple exceptional points, which are known to influence each other strongly. This issue, which has been investigated in toy models [53] and finetuned semiclassical models without local degrees of freedom [54,55], is even less trivial for our quantum many-body model showing an exponential proliferation of exceptional points. Since the number of avoided level crossings in the Hermitian case $\operatorname{Im}(z)=0$ scales as $2^{L}$ and the spectral bandwidth only as $L$, the corresponding real gaps must scale down exponentially like $2^{-L}$. Therefore, making the assumptions (i) and (ii) we can conclude that the exceptional points at the bottom of the distribution approach the real axis like $2^{-L}$.

The assumption (i) can actually be tested by analyzing the braidings between eigenvalues along closed paths taken at different distances from the real axis. The result of this analysis is shown in Fig. 4. Taking paths which start and end at the real axis we can attribute a given order to the swap generated by an exceptional point. As illustrated by Figs. 4(c) and $4(\mathrm{~d})$, this is given by the number of real eigenvalues of the initial Hermitian Hamiltonian, which lie between the two which get swapped. For large system sizes such a path encircles so many exceptional points that only the analysis of the permutation of the levels after closure of the path remains possible. As described in Appendix D, from the cycle structure of the permutation we can extract an average swap order as a function of the distance from the real axis, shown in Fig. 4(b). The bottom of the distribution of exceptional points shown in Fig. 3 lies well within the region where the average swap order is well below 2. Figure 4(b) also indicates a linear growth of the average swap order as a function of the distance from the real axis. This supports the hypothesis that assumption (ii) above not only applies to nearest-neighbor avoided level crossings, but also to eigenvalues which are not neighbors in the Hermitian limit. Indeed, applying this assumption to a pair of levels in the Hermitian spectrum which are separated by a given number of levels $k=0, \ldots, 2^{L}-2$, we would conclude that the typical distance from the real axis of an exceptional point swapping such levels would scale as $d_{k} \sim\left(L / 2^{L}\right) k$ since the spectral bandwidth of the Hermitian Hamiltonian scales as $L$ and the typical level separation scales like $2^{-L}$. This argument reproduces the linear scaling observed in Fig. 4(b). The estimated slope, however, does not agree with our numerics, probably because this rough argument does not take into account the inhomogeneity of the density of states. The above scaling expression for $d_{k}$ also predicts the exceptional point distribution to extend up to values of $\operatorname{Im}(z)$ scaling with $L$, in agreement with Fig. 3(b).

The picture that emerges from these findings is that, while increasing the system size $L$, exceptional points generating a braiding with swapping order $k \ll 2^{L}$ approach the real axis exponentially fast. The fastest class corresponding to the nearest-neighbor swaps $k=0$, defining the bottom of the exceptional point distribution.

\section{DISCUSSION AND OUTLOOK}

An important physical implication of our results concerns the interaction between the levels. In the purely Hermitian picture these interactions take place pairwise between nearest neighbors, which underlies the emergence of random Gaussian spectral statistics and the eigenstate thermalization hypothesis (see discussion at the end of Sec. II). Here, we verify in a generic quantum many-body system the hypothesis that this scenario is a projected manifestation of the more complex phenomenology of eigenvalue braiding through exceptional points in the complex plane. More interestingly, we show that exponentially close to the Hermitian limit actually not only nearest-neighbor levels interact via exceptional points, but also all levels which are separated by a number of levels which is not exponentially large in system size.

Our findings could also offer a useful perspective in the characterization of quantum ergodicity in many-body systems, where also the effect of the openness on generic scenarios for relaxation and thermalization is currently attracting a lot of attention $[67,68]$. Indeed, we can expect the proliferation and accumulation of exceptional points to be generic and peculiar to ergodic systems since, as just spelled above, the level repulsion characterizing chaos in generic quantum many-body systems is deeply connected with the presence of exceptional points. Exceptional points are, on the other hand, expected to appear accidentally at fine-tuned points in regular systems. However, this expectation needs to be supported by a detailed investigation of the comparison between ergodic and nonergodic systems, which we leave to a future study. 
As anticipated at the end of Sec. II, we expect our results to be relevant also for open systems in contact with an environment despite the fact that we consider only a non-Hermitian Hamiltonian. Indeed, in case the coupling to the environment is such that the non-Hermitian component of the Hamiltonian is located in the region of proliferation of exceptional points, only the latter are left as an effect of the openness. Indeed, the proliferation region corresponds to an exponentially small non-Hermitian component, i.e., to exponentially small damping of eigenvalues and correspondingly an exponentially small rate of quantum jumps. We are therefore allowed to neglect the latter, as the post-selection of experimental runs without jumps is going to be exponentially efficient. In view of this argument, interesting questions arise about the possibility of fully isolating an ergodic quantum many-body system from their environment. We leave the exploration of this aspect and its compatibility with experimental observation for future research.

Several other lines of investigation emerge naturally out of this work, like the analysis of spectral statistics in the complex plane and its connection to exceptional points, the investigation of the consequences of the exceptional points proliferation for the dynamics, as well as the extension of this study to disordered and driven systems.

\section{ACKNOWLEDGMENTS}

We would like to thank R. Moessner, F. Jülicher, S. Diehl, P. McClarty, and J. Rau for helpful discussions. This research has been in part supported by DFG through project A07 of SFB 1143 (Project No. 247310070). We furthermore acknowledge PRACE for awarding access to HLRS's Hazel Hen computer based in Stuttgart, Germany under Grant No. 2016153659.

\section{APPENDIX A: ERGODICITY OF THE HERMITIAN MODEL}

Here we show additional data to demonstrate that the model with the parameters used in the main text is indeed fully ergodic in the Hermitian limit. We consider in Fig. 5 two aspects of ergodicity: In the left panel, we compare the statistics of the ratio of adjacent energy levels [61] $r_{n}=\min \left(\left(E_{n+1}-E_{n}\right) /\left(E_{n}-E_{n-1}\right),\left(E_{n}-\right.\right.$ $\left.\left.E_{n-1}\right) /\left(E_{n+1}-E_{n}\right)\right)$ for different system sizes $L$ with the result from random matrix theory in the Gaussian unitary ensemble (GUE), the distributions match very well and strong level repulsion is visible as predicted in random matrix theory.

The second criterion we use to quantify ergodicity is the validity of the eigenstate thermalization hypothesis [56-60] (ETH), which ensures thermalization of the closed system. One condition is that eigenstate expectation values of local operators should become a smooth function of the eigenenergy in the thermodynamic limit $L \rightarrow \infty$, coinciding with the expectation value of the operator in the microcanonical ensemble. In the right panel of Fig. 5, we show results for the operator $\hat{\sigma}_{4}^{x} \hat{\sigma}_{5}^{x}$, which is a term in the Hamiltonian and a part of the energy density, therefore showing a strong positive correlation with the energy eigenvalue. The results are clearly consistent with the eigenstate thermalization hypothesis. We

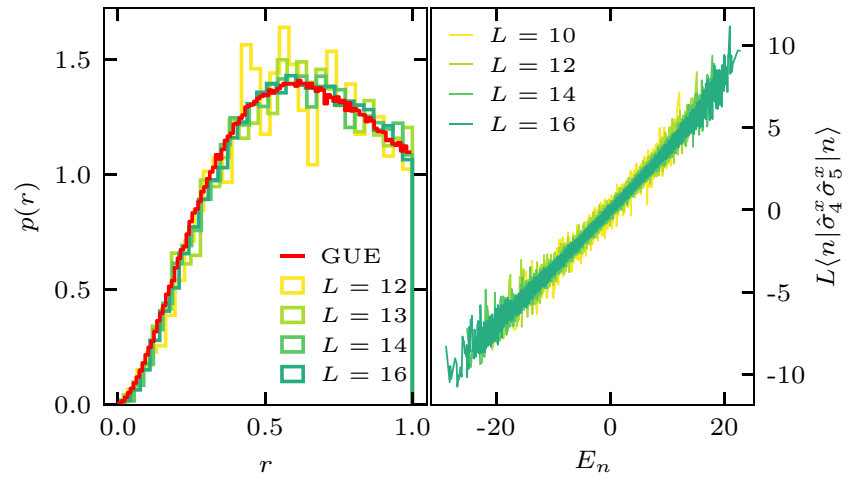

FIG. 5. Left: Distribution of adjacent energy gap ratios $r_{n}=$ $\min \left(\left(E_{n+1}-E_{n}\right) /\left(E_{n}-E_{n-1}\right),\left(E_{n}-E_{n-1}\right) /\left(E_{n+1}-E_{n}\right)\right)$ in comparison with the result for random matrices from the Gaussian unitary ensemble (GUE). Right: eigenstate expectation value $\left\langle n\left|\hat{\sigma}_{4}^{x} \hat{\sigma}_{5}^{x}\right| n\right\rangle$ as a function of eigenenergy $E_{n}$ for different system sizes. Both panels show data for the same parameters as used in the main text and real $z=0.5$, for which the Hamiltonian is Hermitian.

therefore conclude that the model used in the main text is fully ergodic.

\section{APPENDIX B: TRACKING EIGENVALUES}

To extract the number of swaps of eigenvalues, it is necessary to track the evolution of each eigenvalue along a periodic curve $g(\phi):[0,1] \rightarrow \mathbb{C}$ with $g(0)=g(1)$. After closing the curve, the spectra of $\hat{H}(g(0))$ and $\hat{H}(g(1))$ are identical, up to a permutation of the eigenvalues, where each eigenvalue is a continuous function of the curve parameter $\phi$.

It is in general a formidable task to track the evolution of eigenvalues of a quantum many-body Hamiltonian $\hat{H}$ as a function of a (scalar) parameter. Here, we consider a complex parameter $z \in \mathbb{C}$, including the possibility that eigenvalues undergo branch cuts. We are using perturbation theory for this task, which allows us to calculate the derivatives of each eigenvalue with respect to the parameter change along the curve. Comparing the exact new eigenvalues after a small step along the curve with the predictions, we can match each new eigenvalue to the previous ones.

Consider the eigenvalues $E_{n}^{(0)}$ of $\hat{H}\left(z_{0}\right)$ at a point $z_{0}$ in the complex plane. We can predict the eigenvalues of $\hat{H}\left(z_{0}+\right.$ $\epsilon)$ for a small (complex) parameter change $\epsilon$ using nonHermitian perturbation theory.

Let $\left|n_{0}\right\rangle$ be the right eigenvectors of $\hat{H}\left(z_{0}\right)$ with eigenvalue $E_{n}^{(0)}$. Let further $\left|\tilde{n}_{0}\right\rangle$ be the left eigenvectors of $\hat{H}\left(z_{0}\right)$ with eigenvalue $E_{n}^{(0) *}$. The normalization of the eigenvectors can be chosen such that left and right eigenvectors are orthonormal (note that right and left eigenvectors themselves are in general not orthogonal): ${ }^{1}$

$$
\left\langle\tilde{n}_{0} \mid m_{0}\right\rangle=\delta_{n, m} .
$$

\footnotetext{
${ }^{1}$ We note that Lapack routines like zgeev yield a different normalization for the left and right eigenvectors.
} 
Then, perturbation theory for non-Hermitian operators [69] yields

$$
\begin{aligned}
E_{n}\left(z_{0}+\epsilon\right)= & E_{n}\left(z_{0}\right)+\epsilon\left\langle\tilde{n}_{0}\left|\hat{D}\left(z_{0}, \epsilon\right)\right| n_{0}\right\rangle \\
& +\epsilon^{2} \sum_{m \neq n} \frac{\left\langle\tilde{n}_{0}\left|\hat{D}\left(z_{0}, \epsilon\right)\right| m_{0}\right\rangle\left\langle\tilde{m}_{0}\left|\hat{D}\left(z_{0}, \epsilon\right)\right| n_{0}\right\rangle}{E_{m}-E_{n}},
\end{aligned}
$$

with the perturbation $\hat{D}\left(z_{0}, \epsilon\right)=\frac{\hat{H}\left(z_{0}+\epsilon\right)-\hat{H}\left(z_{0}\right)}{\epsilon}$. Due to level repulsion in our system, the denominators $\left(E_{m}-E_{n}\right)$ do not lead to problems if the step size $\epsilon$ is small enough. While degeneracies are possible at exceptional points, the sampled points on the curve did not coincide with exact exceptional points in practice for the system sizes we considered.

This approach allows us to predict for each eigenvalue at $z_{0}$ its change at $z_{0}+\epsilon$, taking into account level crossings and avoided crossings. We fully diagonalize $\hat{H}\left(z_{0}+\epsilon\right)$ and compare the eigenvalues to their predicted locations to attach the labels $n$ to each eigenvalue. This is necessary for two reasons: first, complex eigenvalues do not have a natural ordering and, second, any ordering of eigenvalues used for the labeling would miss swaps and level crossings.

Our procedure depends on a good choice of the step size $\epsilon$ and it is clear that in the proximity of exceptional points very small step sizes have to be used. Therefore, we developed an adaptive method for tracking all eigenvalues along the curve $g(\phi)$, which for each step $g(\phi) \rightarrow g\left(\phi+\delta_{\phi}\right)$ checks if the eigenvalues at $g\left(\phi+\delta_{\phi}\right)$ are sufficiently close to the predictions (compared to the distances between the eigenvalues). If this is not the case, instead of one step $\delta_{\phi}$, two steps of size $\delta_{\phi} / 2$ are carried out. Applying this procedure recursively, the step size is adjusted as needed, allowing a faithful tracking of eigenvalues of very large non-Hermitian Hamiltonians, here up to dimensions of $N=4096$. This allows a precise counting of exceptional points without the need for precisely locating them [70].

\section{APPENDIX C: COUNTING OF EXCEPTIONAL POINTS}

In order to quantify the extreme proliferation of exceptional points with system size visible in Fig. 1, we need a method to count the number of exceptional points in a region in the complex parameter space. Since each exceptional point swaps two eigenvalues of $H(z)$ if a closed path around the exceptional point is followed, we can count the number of exceptional points enclosed by a closed path by counting the number of swaps. Concretely, the number of exceptional points enclosed by a curve $g(\phi):[0,1] \rightarrow \mathbb{C}$ is obtained by tracking all eigenvalues $E_{n}(\phi)$ of $H(g(\phi))$ along the curve and comparing the labels of the eigenvalues of the initial $\phi=0$ and the final point $\phi=1$. Since the spectra are identical, they differ only by a permutation $\pi: E_{n}(0)=E_{\pi(n)}(1)$.

The number of exceptional points encircled by the curve is then equal to the number of transpositions in the permutation $\pi$ :

$$
n_{\mathrm{EP}}=N-n_{\text {cycles }}(\pi) .
$$

\section{APPENDIX D: OBTAINING THE ORDER OF SWAPS}

In Fig. 4, we also analyze which eigenvalues are swapped by exceptional points, when following a path which begins and ends on the real axis in parameter space. For $z \in \mathbb{R}$, the eigenvalues $E_{n}(z)$ of $H(z)$ are real and can be ordered by their magnitude. Following a closed path in the complex parameter plane, we investigate which eigenvalues are swapped by exceptional points as a function of their location relative to the real parameter axis. Since for large systems typical loops $g(\phi)$ encircle always multiple exceptional points, we have to unravel this information from the final permutation of eigenvalues after closing the loop $E_{n}(0)=E_{\pi(n)}(1)$. The permutation $\pi$ is decomposed into cycles of indices, such that indices inside the cycle are shuffled around under multiple applications of the permutation (multiple traversals of the loop), while no other indices are involved in this cycle. Therefore, we can analyze each cycle of the permutation separately, as only the involved eigenvalues are exchanged with each other by the exceptional points enclosed by the path. If only nearest-neighbor eigenvalues are involved in each circle, we call this swaps of order 0 . If on the other hand an exceptional point exchanges eigenvalues separated by $m$ other eigenvalues not in the cycle, we refer to this as a swap of order $m$.
[1] W. D. Heiss, The physics of exceptional points, J. Phys. A: Math. Theor. 45, 444016 (2012).

[2] S.-D. Liang and G.-Y. Huang, Topological invariance and global berry phase in non-Hermitian systems, Phys. Rev. A 87, 012118 (2013).

[3] D. Leykam, K. Y. Bliokh, C. Huang, Y. D. Chong, and F. Nori, Edge Modes, Degeneracies, and Topological Numbers in NonHermitian Systems, Phys. Rev. Lett. 118, 040401 (2017).

[4] W. Hu, H. Wang, P. P. Shum, and Y. D. Chong, Exceptional points in a non-Hermitian topological pump, Phys. Rev. B 95, 184306 (2017).

[5] H. Shen, B. Zhen, and L. Fu, Topological Band Theory for NonHermitian Hamiltonians, Phys. Rev. Lett. 120, 146402 (2018).

[6] F. K. Kunst, E. Edvardsson, J. C. Budich, and E. J. Bergholtz, Biorthogonal Bulk-Boundary Correspondence in Non-Hermitian Systems, Phys. Rev. Lett. 121, 026808 (2018).
[7] Z. Gong, Y. Ashida, K. Kawabata, K. Takasan, S. Higashikawa, and M. Ueda, Topological Phases of Non-Hermitian Systems, Phys. Rev. X 8, 031079 (2018).

[8] V. M. M. Alvarez, J. E. B. Vargas, M. Berdakin, and L. E. F. F. Torres, Topological states of non-Hermitian systems, Eur. Phys. J.: Spec. Top. 227, 1295 (2018).

[9] S. Yao and Z. Wang, Edge States and Topological Invariants of Non-Hermitian Systems, Phys. Rev. Lett. 121, 086803 (2018).

[10] Y. Xiong, Why does bulk boundary correspondence fail in some non-Hermitian topological models, J. Phys. Commun. 2, 035043 (2018).

[11] A. Ghatak and T. Das, New topological invariants in nonHermitian systems, J. Phys.: Condens. Matter 31, 263001 (2019).

[12] D. S. Borgnia, A. J. Kruchkov, and R.-J. Slager, Non-Hermitian boundary modes, arXiv:1902.07217. 
[13] M. S. Rudner and L. S. Levitov, Topological Transition in A Non-Hermitian Quantum Walk, Phys. Rev. Lett. 102, 065703 (2009).

[14] C. Yuce, Topological phase in a non-Hermitian pt symmetric system, Phys. Lett. A 379, 1213 (2015).

[15] S. Malzard, C. Poli, and H. Schomerus, Topologically Protected Defect States in Open Photonic Systems with Non-Hermitian Charge-Conjugation and Parity-Time Symmetry, Phys. Rev. Lett. 115, 200402 (2015).

[16] T. E. Lee, Anomalous Edge State in a Non-Hermitian Lattice, Phys. Rev. Lett. 116, 133903 (2016).

[17] P. San-Jose, J. Cayao, E. Prada, and R. Aguado, Majorana bound states from exceptional points in non-topological superconductors, Sci. Rep. 6, 21427 (2016).

[18] H. Menke and M. M. Hirschmann, Topological quantum wires with balanced gain and loss, Phys. Rev. B 95, 174506 (2017).

[19] C. Yin, H. Jiang, L. Li, R. Lü, and S. Chen, Geometrical meaning of winding number and its characterization of topological phases in one-dimensional chiral non-hermitian systems, Phys. Rev. A 97, 052115 (2018).

[20] S. Lieu, Topological phases in the non-Hermitian Su-SchriefferHeeger model, Phys. Rev. B 97, 045106 (2018).

[21] V. M. Martinez Alvarez, J. E. Barrios Vargas, and L. E. F. Foa Torres, Non-Hermitian robust edge states in one dimension: Anomalous localization and eigenspace condensation at exceptional points, Phys. Rev. B 97, 121401(R) (2018).

[22] R. Wang, X. Z. Zhang, and Z. Song, Dynamical topological invariant for the non-Hermitian rice-mele model, Phys. Rev. A 98, 042120 (2018).

[23] S. Lieu, Topological symmetry classes for non-hermitian models and connections to the bosonic Bogoliubov-de Gennes equation, Phys. Rev. B 98, 115135 (2018).

[24] C. H. Lee and R. Thomale, Anatomy of skin modes and topology in non-Hermitian systems, Phys. Rev. B 99, 201103(R) (2019).

[25] B. Dóra, M. Heyl, and R. Moessner, The Kibble-Zurek mechanism at exceptional points, Nat. Commun. 10, 2254 (2019).

[26] K. Esaki, M. Sato, K. Hasebe, and M. Kohmoto, Edge states and topological phases in non-hermitian systems, Phys. Rev. B 84, 205128 (2011).

[27] Y. Xu, S.-T. Wang, and L.-M. Duan, Weyl Exceptional Rings in A Three-Dimensional Dissipative Cold Atomic Gas, Phys. Rev. Lett. 118, 045701 (2017).

[28] J. Carlström and E. J. Bergholtz, Exceptional links and twisted fermi ribbons in non-Hermitian systems, Phys. Rev. A 98, 042114 (2018).

[29] R. A. Molina and J. González, Surface and 3d Quantum Hall Effects from Engineering of Exceptional Points in Nodal-Line Semimetals, Phys. Rev. Lett. 120, 146601 (2018).

[30] S. Yao, F. Song, and Z. Wang, Non-Hermitian Chern Bands, Phys. Rev. Lett. 121, 136802 (2018).

[31] T. Yoshida, R. Peters, and N. Kawakami, Non-Hermitian perspective of the band structure in heavy-fermion systems, Phys. Rev. B 98, 035141 (2018).

[32] J. C. Budich, J. Carlström, F. K. Kunst, and E. J. Bergholtz, Symmetry-protected nodal phases in non-Hermitian systems, Phys. Rev. B 99, 041406(R) (2019).

[33] T. Yoshida, R. Peters, N. Kawakami, and Y. Hatsugai, Symmetry-protected exceptional rings in two-dimensional correlated systems with chiral symmetry, Phys. Rev. B 99, 121101(R) (2019).

[34] Z. Yang and J. Hu, Non-Hermitian hopf-link exceptional line semimetals, Phys. Rev. B 99, 081102(R) (2019).

[35] H. Wang, J. Ruan, and H. Zhang, Non-hermitian nodal-line semimetals with an anomalous bulk-boundary correspondence, Phys. Rev. B 99, 075130 (2019).

[36] P. A. McClarty and J. G. Rau, Non-Hermitian topology of spontaneous magnon decay, Phys. Rev. B 100, 100405(R) (2019).

[37] I. Rotter, A non-Hermitian hamilton operator and the physics of open quantum systems, J. Phys. A: Math. Theor. 42, 153001 (2009).

[38] Y. Choi, S. Kang, S. Lim, W. Kim, J.-R. Kim, J.-H. Lee, and K. An, Quasieigenstate Coalescence in An Atom-Cavity Quantum Composite, Phys. Rev. Lett. 104, 153601 (2010).

[39] M. Brandstetter, M. Liertzer, C. Deutsch, P. Klang, J. Schöberl, H. E. Türeci, G. Strasser, K. Unterrainer, and S. Rotter, Reversing the pump dependence of a laser at an exceptional point, Nat. Commun. 5, 4034 (2014).

[40] T. Gao, E. Estrecho, K. Y. Bliokh, T. C. H. Liew, M. D. Fraser, S. Brodbeck, M. Kamp, C. Schneider, S. Höfling, Y. Yamamoto, F. Nori, Y. S. Kivshar, A. G. Truscott, R. G. Dall, and E. A. Ostrovskaya, Observation of non-hermitian degeneracies in a chaotic exciton-polariton billiard, Nature (London) 526, 554 (2015).

[41] J. M. Zeuner, M. C. Rechtsman, Y. Plotnik, Y. Lumer, S. Nolte, M. S. Rudner, M. Segev, and A. Szameit, Observation of A Topological Transition in the Bulk of A Non-Hermitian System, Phys. Rev. Lett. 115, 040402 (2015).

[42] C. Poli, M. Bellec, U. Kuhl, F. Mortessagne, and H. Schomerus, Selective enhancement of topologically induced interface states in a dielectric resonator chain, Nat. Commun. 6, 6710 (2015).

[43] J. Doppler, A. A. Mailybaev, J. Böhm, U. Kuhl, A. Girschik, F. Libisch, T. J. Milburn, P. Rabl, N. Moiseyev, and S. Rotter, Dynamically encircling an exceptional point for asymmetric mode switching, Nature (London) 537, 76 (2016).

[44] H. Xu, D. Mason, L. Jiang, and J. G. E. Harris, Topological energy transfer in an optomechanical system with exceptional points, Nature (London) 537, 80 (2016)

[45] B. Peng, Ş. K. Özdemir, M. Liertzer, W. Chen, J. Kramer, H. Y1lmaz, J. Wiersig, S. Rotter, and L. Yang, Chiral modes and directional lasing at exceptional points, Proc. Natl. Acad. Sci. USA 113, 6845 (2016).

[46] W. Chen, Ş. K. Özdemir, G. Zhao, J. Wiersig, and L. Yang, Exceptional points enhance sensing in an optical microcavity, Nature (London) 548, 192 (2017).

[47] M. A. Bandres, S. Wittek, G. Harari, M. Parto, J. Ren, M. Segev, D. N. Christodoulides, and M. Khajavikhan, Topological insulator laser: Experiments, Science 359, eaar4005 (2018).

[48] H. Zhou, C. Peng, Y. Yoon, C. W. Hsu, K. A. Nelson, L. Fu, J. D. Joannopoulos, M. Soljačić, and B. Zhen, Observation of bulk fermi arc and polarization half charge from paired exceptional points, Science 359, 1009 (2018).

[49] A. Cerjan, S. Huang, K. P. Chen, Y. Chong, and M. C. Rechtsman, Experimental realization of a Weyl exceptional ring, Nat. Photonics 13, 623 (2019).

[50] L. Xiao, X. Zhan, Z. H. Bian, K. K. Wang, X. Zhang, X. P. Wang, J. Li, K. Mochizuki, D. Kim, N. Kawakami, W. Yi, H. Obuse, B. C. Sanders, and P. Xue, Observation of topological 
edge states in parity-time-symmetric quantum walks, Nat. Phys. 13, 1117 (2017).

[51] Y. Wu, W. Liu, J. Geng, X. Song, X. Ye, C.-K. Duan, X. Rong, and J. Du, Observation of parity-time symmetry breaking in a single-spin system, Science 364, 878 (2019).

[52] R. Hanai and P. B. Littlewood, Critical fluctuations at a manybody exceptional point, arXiv:1908.03243.

[53] W. D. Heiss and A. L. Sannino, Avoided level crossing and exceptional points, J. Phys. A: Math. Gen. 23, 1167 (1990).

[54] W. D. Heiss, F. G. Scholtz, and H. B. Geyer, The largeNbehaviour of the lipkin model and exceptional points, J. Phys. A: Math. Gen. 38, 1843 (2005).

[55] P. Stránský, M. Dvořák, and P. Cejnar, Exceptional points near first- and second-order quantum phase transitions, Phys. Rev. E 97, 012112 (2018).

[56] J. M. Deutsch, Quantum statistical mechanics in a closed system, Phys. Rev. A 43, 2046 (1991).

[57] M. Srednicki, Chaos and quantum thermalization, Phys. Rev. E 50, 888 (1994).

[58] M. Rigol, V. Dunjko, and M. Olshanii, Thermalization and its mechanism for generic isolated quantum systems, Nature (London) 452, 854 (2008).

[59] L. D’Alessio, Y. Kafri, A. Polkovnikov, and M. Rigol, From Quantum Chaos and Eigenstate Thermalization to Statistical Mechanics and Thermodynamics, Adv. Phys. 65, 239 (2016).

[60] F. Borgonovi, F. M. Izrailev, L. F. Santos, and V. G. Zelevinsky, Quantum chaos and thermalization in isolated systems of interacting particles, Phys. Rep. 626, 1 (2016).
[61] V. Oganesyan and D. A. Huse, Localization of interacting fermions at high temperature, Phys. Rev. B 75, 155111 (2007).

[62] C. Gardiner, P. Zoller, and P. Zoller, Quantum Noise: A Handbook of Markovian and Non-Markovian Quantum Stochastic Methods with Applications to Quantum Optics, Vol. 56 (Springer, New York, 2004).

[63] H.-P. Breuer et al., The Theory of Open quantum systems (Oxford University Press, Oxford, 2002).

[64] A. J. Daley, Quantum trajectories and open many-body quantum systems, Adv. Phys. 63, 77 (2014).

[65] T. E. Lee and C.-K. Chan, Heralded Magnetism in NonHermitian Atomic Systems, Phys. Rev. X 4, 041001 (2014).

[66] T. Katō, Perturbation Theory for Linear Operators, Classics in Mathematics (Springer, Berlin, 1995).

[67] E. Levi, M. Heyl, I. Lesanovsky, and J. P. Garrahan, Robustness of Many-Body Localization in the Presence of Dissipation, Phys. Rev. Lett. 116, 237203 (2016).

[68] H. P. Lüschen, P. Bordia, S. S. Hodgman, M. Schreiber, S. Sarkar, A. J. Daley, M. H. Fischer, E. Altman, I. Bloch, and U. Schneider, Signatures of Many-Body Localization in a Controlled Open Quantum System, Phys. Rev. X 7, 011034 (2017).

[69] M. M. Sternheim and J. F. Walker, Non-Hermitian Hamiltonians, Decaying States, and Perturbation Theory, Phys. Rev. C 6, 114 (1972).

[70] M. Feldmaier, J. Main, F. Schweiner, H. Cartarius, and G. Wunner, Rydberg systems in parallel electric and magnetic fields: an improved method for finding exceptional points, J. Phys. B: At., Mol. Opt. Phys. 49, 144002 (2016). 